\title{
Comparative Fluoride Release and Antimicrobial Analysis of Commercial and Experimental Bioactive Glass/Nano-Oxide-Based Dentifrices
}

\author{
Hashmat Gul ${ }^{1} \quad$ Maleeha Nayyer ${ }^{1} \quad$ Mehreen Gilani $^{2} \quad$ Nadia Aman $^{3} \quad$ Azad Ali Azad $^{4}$ \\ Asma Tufail Shah ${ }^{5}$ Aqif Anwar Chaudhry ${ }^{5}$ Muhammad Kaleem ${ }^{1} \quad$ Abdul Samad Khan ${ }^{6, \odot}$
}

${ }^{1}$ Department of Dental Materials, Army Medical College, National University of Medical Sciences, Rawalpindi, Pakistan

2Department of Microbiology, Army Medical College, National University of Medical Sciences, Rawalpindi, Pakistan

${ }^{3}$ Department of Operative Dentistry, Foundation University Medical College, Islamabad, Pakistan

${ }^{4}$ Department of Prosthodontics, Armed Forces Institute of Dentistry, National University of Medical Sciences, Rawalpindi, Pakistan

5 Interdisciplinary Research Center in Biomedical Materials, COMSATS University Islamabad Lahore Campus, Lahore, Pakistan

${ }^{6}$ Department of Restorative Dental Sciences, College of Dentistry, Imam Abdulrahman Bin Faisal University, Dammam, Saudi Arabia

Eur J Dent 2020;14:38-44

\author{
Address for correspondence Abdul Samad Khan, BDS, MSc, PhD, \\ Department of Restorative Dental Sciences, College of Dentistry, \\ Imam Abdulrahman Bin Faisal University, Dammam 31441, \\ Saudi Arabia (e-mail: akhan@iau.edu.sa).
}

\begin{abstract}
Keywords

- toothpaste

- nanoparticles

- bioactive glass

- zinc oxide

- fluoride release

- antibacterial

Objectives The objectives were to measure fluoride release and assess the antimicrobial behavior of fluoride-doped nano bioactive glass (F-nBG) and nano zinc oxide (ZnO)-enriched novel dentifrices.

Materials and Methods Experimental dentifrices were synthesized by incorporating $\mathrm{ZnO}$ nanoparticles and $\mathrm{F}-\mathrm{nBG}$ ( $1.5 \mathrm{wt} \%$ and $4 \mathrm{wt} \%$ ) as active ingredients. The fluoride release behavior of suspensions and elutes of samples were analyzed by ion selective electrode. Antimicrobial activity and minimum bactericidal concentration against Streptococcus mutans and Lactobacillus casei were evaluated. Microbial stability against contamination was also assessed by a challenge test.

Results The fluoride release behavior of experimental dentifrices was higher than that of commercial dentifrices and was dependent on filler loading. The fluoride release was more from suspensions than elutes. Zones of inhibition (ZOls) and minimum bactericidal concentration values for novel dentifrices showed direct proportionality with filler loading, and effectiveness was exhibited against both strains. Experimental dentifrices exhibited effective antibacterial potential, which could possibly be due to release of sufficient fluoride and zinc ions in aqueous media from $\mathrm{F}-\mathrm{nBG}$ and $\mathrm{ZnO}$ present in their formulations.

Conclusion Combination of $\mathrm{F}-\mathrm{nBG}$ and $\mathrm{ZnO}$ may provide a multi-benefit approach for simultaneously treating early white spot lesions, reducing bacterial growth, and providing core plaque control.
\end{abstract}

\section{Introduction}

Brushing teeth with fluoridated toothpaste has been widely practiced worldwide for dental caries prevention, as it removes biofilm mechanically, which adds to the therapeutic effects of fluoride. Other ingredients for functions such as whitening, antiplaque, antibacterial, antitartar, and erosive prevention can be added. ${ }^{1}$ Availability and stability of fluoride in dentifrices are basic requirements for effectiveness against tooth decay. ${ }^{2,3}$ Fluoride ions can inhibit 
the production of bacterial acids in dental plaque due to the influx of hydrogen fluoride into bacterial cells, and the dissociation to the $\mathrm{H}^{+}$and F-ions in the cytoplasm. ${ }^{4}$ For caries control, dentifrice formulation should contain a minimum of 1000 parts per million (ppm) fluoride, which must be in a soluble form to impart the anticaries effect. ${ }^{5,6}$ The factors which control the soluble fluoride content in dentifrice are its composition (i.e., fluoride source) and storage conditions (i.e., temperature and aging). It is reported that low-pH toothpastes containing 500 to $550 \mathrm{ppm}$ fluoride showed similar results on enamel demineralization as the one containing 1100 ppm fluoride. ${ }^{7}$

Dentifrice composition plays a crucial role in disabling free fluoride ions, which may lead to creation of a low-solubility product with a diminished anticaries outcome. ${ }^{2,3}$ Currently, there are no regulations to specify how much of total fluoride should be maintained in a dentifrice formulation. ${ }^{5}$ There has been a growing interest in the use of "smart" bioactive materials (i.e., amorphous calcium phosphate, hydroxyapatite, bioactive glass, etc.) for tooth remineralization. It is reported that the remineralization effect of nano-hydroxyapatite crystals is generally limited to the surface of the initial carious lesion. ${ }^{8}$ Bioactive glass is one such material being considered as a novel dental material with remineralization potential. Some in vitro studies have shown that bioactive glass-containing dentifrices minimized dentin hypersensitivity by dentinal tubule occlusion and apatite formation. ${ }^{9} 10$

Recently, fluoride-doped bioactive glass nanoparticles (F-nBG) have been synthesized by our group, and the longterm fluoride release behavior of F-nBG and its effect on $\mathrm{pH}$ was evaluated. ${ }^{11}$ Zinc oxide ( $\mathrm{ZnO}$ ) powder has been added as a preservative in dentifrices, which, in aqueous suspension, not only inhibits dentin demineralization but also induces antimicrobial action by yielding zinc ions $\left(\mathrm{Zn}^{2+}\right)$ and reactive oxygen species. ${ }^{12-14}$ The purpose of this study was to assess and compare the antimicrobial efficacy and fluoride release behavior from the suspensions and elutes of experimental dentifrices and a commercial dentifrice.

\section{Materials and Methods}

In this study, all chemicals were analytical grade. Initially, basic ingredients were mixed in optimized ratios and allowed to mix homogeneously. Then $1.5 \mathrm{wt} \%$ titanium dioxide (Sigma Aldrich; St. Louis, MO, United States) and 3 wt\% zinc oxide nanoparticles were added in increments to avoid agglomeration. The $\mathrm{ZnO}$ nanoparticles were synthesized by our group as described previously. ${ }^{15}$ The scanning electron microscopy (SEM) image and X-ray diffraction (XRD) pattern of prepared $\mathrm{ZnO}$ nanoparticles are shown in - Fig. 1. The morphological pattern showing the nanostructure of prepared $\mathrm{ZnO}$ and the diffractogram is in accordance with JCPDS N 00-036-1451 (standard X-ray peaks of zinc oxide). F-nBG (5\% mol. concentration of $\mathrm{F}^{-}$in $\mathrm{F}-\mathrm{BG}$ ) was used in this study and incorporated in experimental dentifrices in two ratios: $1.5 \%$ and $4 \% \mathrm{wt} / \mathrm{wt}$. Colgate Cavity Protection (Colgate-Palmolive, Guildford, UK) was used as the control. The composition of both the control and the experimental dentifrices is given in -Table 1 .

\section{Fluoride Release Analysis}

The fluoride research protocol used in this study was modified from the one suggested by Pearce. ${ }^{16}$ The study was performed in quadruplicates. From each group, a $100 \mathrm{mg}$ dentifrice sample was homogenized in $10 \mathrm{~mL}$ deionized water to form dentifrice suspensions, from which half of the suspension was split in two centrifuge tubes, each containing $2.5 \mathrm{~mL}$ suspension. The remaining half dentifrice suspension was centrifuged (Hettich EBA 20; Hettich, Tuttlingen, Germany) at $6000 \mathrm{rpm}$ for $10 \mathrm{~min}(2461 \times \mathrm{g})$ to remove the insoluble fluoride bound to filler particles to extract elute into two centrifuge tubes, each containing $2.5 \mathrm{~mL}$ of elute. The same procedure was repeated for all dentifrice groups. Then $2.5 \mathrm{~mL}$ of $2 \mathrm{M} \mathrm{HCl}$ (BDH Chemicals; Hull, East Yorkshire, UK) was added to all the centrifuge tubes containing the dentifrice elute and suspension samples, which were then conditioned at $45^{\circ} \mathrm{C}$ for 1 hour followed by an addition of $5 \mathrm{~mL}$ of $1 \mathrm{M} \mathrm{NaOH}$ (Sigma Aldrich) and $1 \mathrm{~mL} \mathrm{TISAB}$ III reagent (Hanna Instruments; Woonsocket, Rhode Island, United States). Finally, the samples were analyzed using precalibrated ion selective electrode (ISE) potentiometry (Hanna HI3222 pH/ISE meter and fluoride electrode; Hanna Instruments, Woonsocket, Rhode Island, United States).

\section{Antimicrobial Susceptibility Test}

For the antimicrobial susceptibility test, freeze-dried microbial strains Streptococcus mutans (ATCC-25175) and Lactobacillus casei (ATCC-393) were obtained from American Type Culture Collection (ATCC; Manassas, VA, United States). Dehydrated culture media was obtained from Oxoid (Basingstoke, Hampshire, England) and Merck Millipore (Darmstadt, Germany). Microorganisms were revived from freeze-dried vials in their respective culture media-MRS (de Man Rogosa) broth for $L$. casei and BHI (Brain Heart Infusion) broth for S. mutans-as per American type culture collection (ATCC) guidelines. For the test procedure, $2 \mathrm{~g}$ of selected dentifrice (-Table 1) was mixed in $2 \mathrm{~mL}$ of sterile deionized water, and this yielded a 1:1 dilution. The same procedure was applied to prepare three dilutions of each dentifrice in the ratios of $1: 1,1: 2$, and $1: 4$, respectively. Dentifrice slurries were vortexed in an electric shaker (Heidolph REAX 2000; Gemini BV Laboratory, DG Apeldoorn, the Netherlands). For inoculum preparation, three to five well-isolated, similar colonies were selected and transferred to tubes containing $5 \mathrm{~mL}$ of $0.9 \%$ saline. The suspensions were then adjusted to a density equivalent to 0.5 standard of the McFarland Turbidity Scale (DensiCHEK plus; bioMérieux, NC, United States). Within $15 \mathrm{~min}$ of the preparation of the adjusted inoculum suspensions, it was swabbed evenly over the entire surface of the respective agar plates of prepared media to create a bacterial lawn.

\section{Agar Well-Diffusion Assay}

A sterile cork borer was used to create four wells in each of the agar plates. The punched agar circles were picked with a sterile needle and removed from the plates. The wells were $8 \mathrm{~mm}$ in diameter and $4 \mathrm{~mm}$ in depth and were placed at distances of $20 \mathrm{~mm}$ in each of the plates. Then, $0.2 \mathrm{~mL}$ of the three 
Table 1 Grouping and composition of dentifrices used in the study

\begin{tabular}{|c|c|c|c|c|}
\hline Dentifrice & Abrasive & $\begin{array}{l}\text { Other ingredients } \\
(\% \mathrm{wt})\end{array}$ & $\begin{array}{l}\text { Fluoride agent } \\
\text { Percentage }\end{array}$ & Group name \\
\hline $\begin{array}{l}\text { Colgate cavity protection } \\
\text { (Colgate-Palmolive, } \\
\text { Guildford, UK) } \\
\text { Lot-number: } 10141222\end{array}$ & $\begin{array}{l}\text { Calcium } \\
\text { carbonate } \\
\left(\mathrm{CaCO}_{3}\right)\end{array}$ & $\begin{array}{l}\text { Water } \\
\text { Glycerin } \\
\text { Sodium lauryl sulfate } \\
\text { Sodium saccharin } \\
\text { Cellulose gum } \\
\text { Flavoring agent } \\
\text { Tetrasodium pyrophosphate }\end{array}$ & $\begin{array}{l}\text { SMFP } \\
0.76 \mathrm{w} / \mathrm{w} \\
(0.15 \mathrm{w} / \mathrm{v})\end{array}$ & $C D$ \\
\hline \multirow[t]{2}{*}{ Experimental dentifrices } & \multirow{2}{*}{$\begin{array}{l}\text { Calcium car- } \\
\text { bonate } \\
\left(\mathrm{CaCO}_{3}\right) 27 \% \mathrm{wt}\end{array}$} & \multirow{2}{*}{$\begin{array}{l}\text { Water 36\% } \\
\text { Glycerol 32\% } \\
\text { Sodium benzoate 1\% } \\
\text { Methyelcellulose 1\% } \\
\text { Flavoring agent 1\% } \\
\text { Sodium lauryl sulfate 2\% } \\
\text { Water 36\% } \\
\text { Titanium dioxide }\left(\mathrm{TiO}_{2}\right) \text { 1.5\% } \\
\text { Zinc oxide ( } \mathrm{ZnO}) \\
\text { nanoparticles 3\% }\end{array}$} & $\begin{array}{l}\text { F-nBG with } 5 \% \text { mol fluoride } \\
1.5 \% \text { F-nBG }(w / w)\end{array}$ & ED1 \\
\hline & & & $\begin{array}{l}\text { F-nBG with } 5 \% \text { mol fluoride } \\
4 \% \text { F-nBG }(w / w)\end{array}$ & ED2 \\
\hline
\end{tabular}

Abbreviation: SMFP, sodium monofluorophosphate.
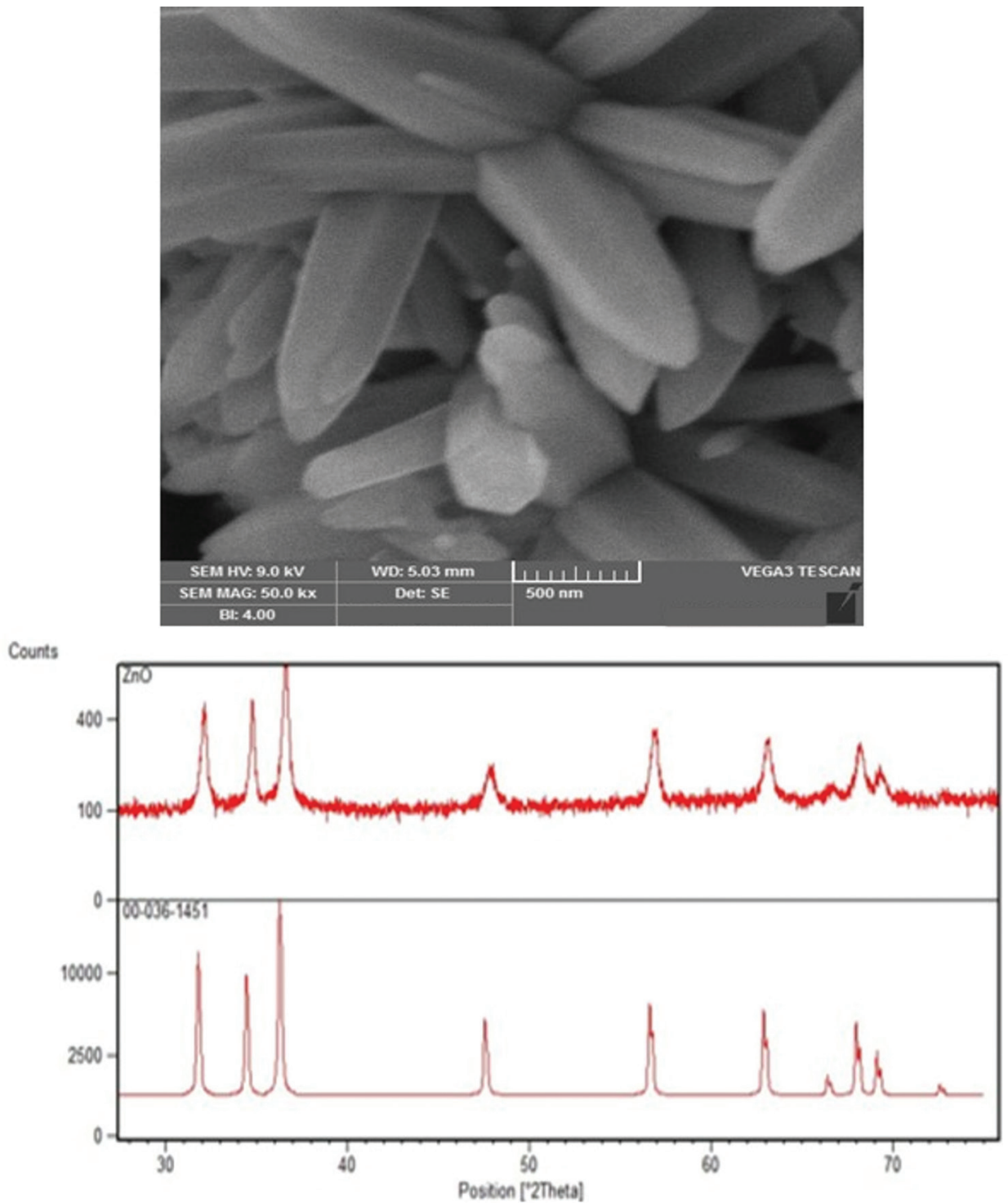

Fig. 1 SEM image and X-ray diffractogram of synthesized zinc oxide. SEM, scanning electron microscope. 
dilutions (1:1, 1:2, and 1:4) of each dentifrice and a control containing deionized water were poured into separate wells in each agar plate using a micropipette (Genex Beta; Guangdong China). The pH of prepared, solid agar mediums used for this study was similar to the physiological $\mathrm{pH}$ of plaque. ${ }^{17}$

All the plates were made in triplicate, and S. mutans was incubated in both aerobic and anaerobic conditions and tested on Muellar-Hinton agar plates. In the case of $L$. casei, the sets of triplicate were tested in microaerophilic as well as predominant anaerobic conditions on chocolate blood agar plates (Gas-Pak sachet-Oxoid AnaeroGen; Thermo Fisher Scientific, Bartlesville, OK, United States).

\section{Determination of Minimum Bactericidal Concentration}

Susceptibility break points are expressed as minimum inhibitory concentration (MIC) and/or minimum bactericidal concentration $(\mathrm{MBC})$ using appropriate dilution methods, whereby two-fold dilutions of the experimental and commercial groups were prepared as 1:1, 1:2, 1:4, 1:8, and 1:16 ( $2 \mathrm{~g} / 2 \mathrm{~mL}$ yielded a 1:1 dilution). MBC was determined using modified broth dilution method for dentifrices. ${ }^{18}$ Then, $1 \mathrm{~mL}$ of each dilution of dentifrice was mixed with $1 \mathrm{~mL}$ of respective broth to yield a defined volume of 2 $\mathrm{mL}$ in each of the five test tubes. A test tube without dentifrice, containing $2 \mathrm{~mL}$ of deionized water for each bacterial strain, was used as control.

All the test tubes, including controls, were inoculated using a sterile wire loop. The test tubes inoculated with $S$. mutans were incubated aerobically for 24 hours at $37^{\circ} \mathrm{C}$, whereas the test tubes inoculated with $L$. casei were incubated for
48 hours at $37^{\circ} \mathrm{C}$. To ensure reproducibility, this step was repeated twice. Following incubation, all test tubes were sub-cultured to Mueller-Hinton agar (S. mutans) and chocolate blood agar plates (L. casei) in duplicate. The plate which showed no visible growth was taken as MBC and reported in $\mathrm{g} / \mathrm{mL}$ of the corresponding dilution.

\section{Statistical Analysis}

SPSS version 22 (IBM; Armonk, New York, United States) was used to calculate the means and standard deviations of the groups along with differences within and between the groups by one-way ANOVA, and a posthoc Tukey's test was used for pairwise comparison between groups.

\section{Results}

\section{Fluoride Release Analysis}

The fluoride release in ppm for both the elutes and the suspensions of all the study groups are presented in - Fig. 2. Among elutes of the dentifrices, maximum mean fluoride release ( \pm standard deviation) was detected in ED2 (13.22 $\pm 0.09 \mathrm{ppm})$, followed by ED1 (9.74 $\pm 0.05 \mathrm{ppm})$, and the control dentifrice $(6.28 \pm 0.05 \mathrm{ppm})$. Among suspensions of the study dentifrices, the maximum mean fluoride release was exhibited by ED2 (14.20 $\pm 0.09 \mathrm{ppm})$, followed by ED1 (10.07 $\pm 0.09 \mathrm{ppm})$, and the control group $(6.63 \pm 0.05 \mathrm{ppm})$. In both elutes and suspensions of experimental dentifrices, a rise in mean fluoride release was observed with a rise in F-nBG filler content. The posthoc Tukey's test showed a statistically significant difference

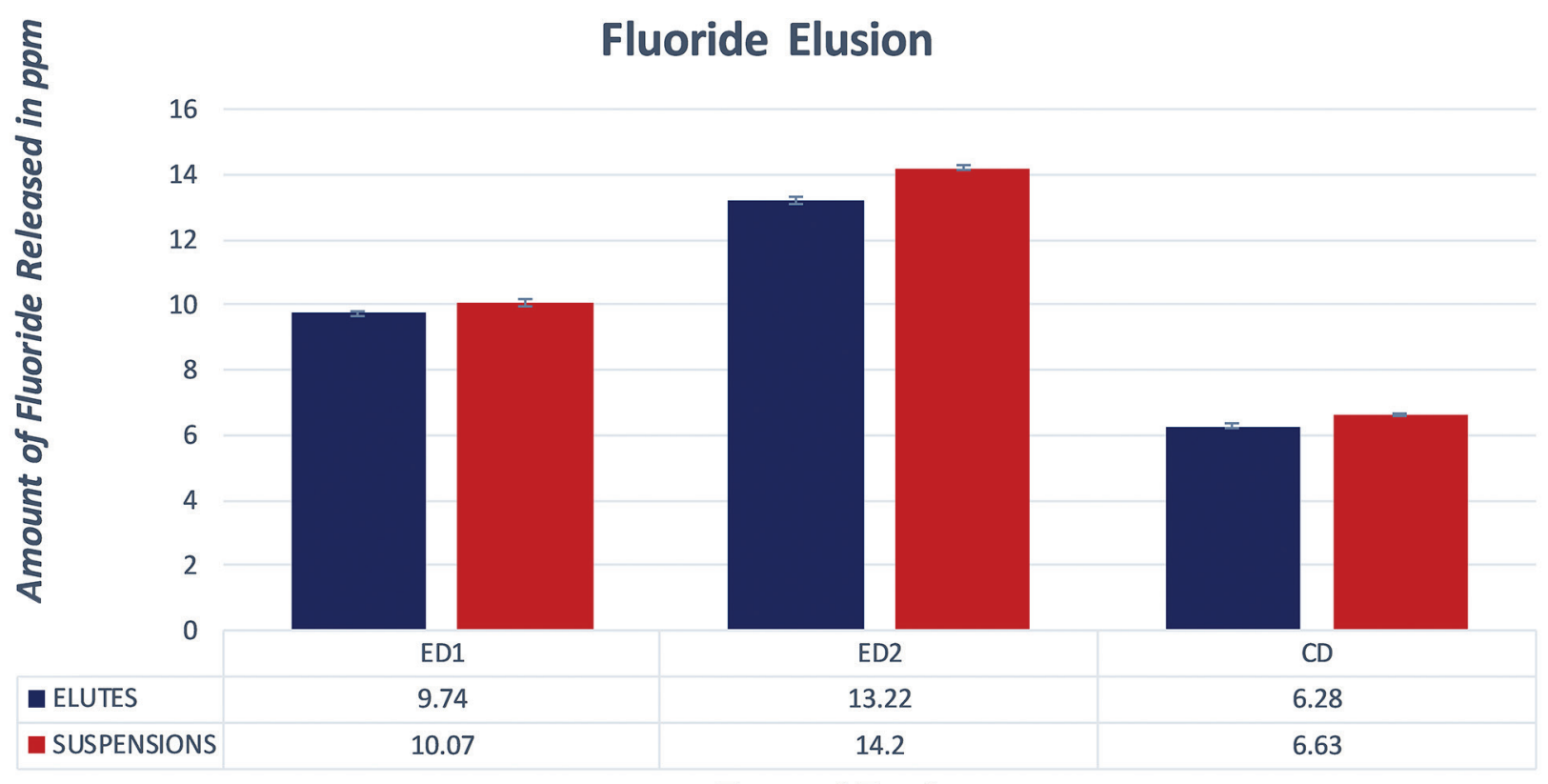

Types of Toothpastes

Fig. 2 Mean fluoride elusion values with standard deviations (error bars) in the elutes and suspensions of the experimental dentifrices. 
between the mean fluoride release of elutes of all the study dentifrice groups and between the mean fluoride release of suspensions of all study dentifrice groups $(p=0.000)$.

\section{Antimicrobial Analysis \\ Antimicrobial Susceptibility Test: Agar Well Diffusion Assay}

Following incubation, resultant zones of inhibition were observed on the agar plates. The clear and circular halos surrounding the wells were observed. The zone of inhibition against $S$. mutans appeared after 24 hours, and the inhibition zone against $L$. case $i$ appeared after 48 hours. The inhibition zones increased in a dose-dependent manner for the dilutions $1: 1,1: 2$, and $1: 4$ of dentifrice slurries. The negative control wells containing deionized water produced no observable inhibitory effect for any of the bacteria in each of the triplicate sets of agar plates. The assessment of antibacterial potential against caries-causing odonto-pathogens was done by recording the diameters of zones of inhibition (ZOIs) (mm) using a Vernier caliper (-Table 2). One-way AVOVA (analysis of variance) revealed that only ED1 and CD indicated statistical significance $(p<0.05)$. Pair-wise comparisons using a posthoc Tukey's test revealed differences between the concentration groups. ED1 and ED2 were statistically insignificant $(p>0.05)$ for both bacterial strains under all conditions.

\section{Determination of Minimum Bactericidal Concentration} ED2 showed increased effectiveness and was bactericidal (S. mutans) even at the dilution of 1:16, similar to CD. In the case of $L$. casei, ED1 was effective up to a dilution 1:4 only and growth was observed on plates at a dilution of 1:8. This finding also corresponded with the smallest mean ZOIs observed for ED1 against L. casei using agar well-diffusion assay (-Table 2). The remaining dentifrices were bactericidal (L. casei) up to a dilution of 1:8. For both bacterial strains, marked growth was observed on the plated controls.

\section{Discussion}

In the current study, evaluation of the proportions of F-nBG fillers in dentifrices that yield maximum fluoride elusion among novel dentifrices was performed. Upon increasing the F-nBG filler content in experimental dentifrice groups, an increase in fluoride release rates in both elutes and suspensions of dentifrices was observed, whereas a direct proportionality of fluoride release rate with the F-nBG filler loading in novel dentifrices was observed.

Dentifrices are used in suspension form during tooth brushing procedures; therefore, the fluoride elusion in dentifrice suspensions is of greater clinical significance than the fluoride elusion in the dentifrice elutes. However, no remarkable difference was observed in fluoride release values of elutes and suspensions of the same group. This may possibly be due to instant release of fluoride ions upon dissolution of fluoride compounds used in experimental dentifrices in aqueous media. Regarding the bactericidal effect of fluoride, it is reported that fluoride release of $20 \mathrm{ppm}$ exhibits direct bactericidal action. ${ }^{19}$ However, due to the buffering action of saliva, this amount drops and may not be as relevant clinically. The elutes and suspension of ED2 showed $13.72 \mathrm{ppm}$ and $14.20 \mathrm{ppm}$ and close to the required amount.

In a previous study conducted by our group, F-nBG particles with $5 \mathrm{wt} \%$ fluoride showed burst fluoride release for the initial 9 days. ${ }^{11}$ As dentifrices are used to brush teeth for only a few minutes, therefore, the burst fluoride release by F-nBG filler particles with $5 \mathrm{wt} \%$ fluoride is of clinical significance in dentifrices. Addition of fluoride to bioactive glass

Table 2 Mean diameter and SDs of ZOI against microbial strains

\begin{tabular}{|c|c|c|c|c|c|c|c|}
\hline \multicolumn{8}{|c|}{ ZOls against S. mutans } \\
\hline \multirow{2}{*}{\multicolumn{2}{|c|}{ Experimental }} & \multicolumn{2}{|l|}{$\begin{array}{l}\text { Mean } \pm \text { SD } \\
\text { Dilution 1:1 }\end{array}$} & \multicolumn{2}{|c|}{$\begin{array}{l}\text { Mean } \pm \text { SD } \\
\text { Dilution } 1: 2\end{array}$} & \multicolumn{2}{|l|}{$\begin{array}{l}\text { Mean } \pm \text { SD } \\
\text { Dilution 1:4 }\end{array}$} \\
\hline & & \multirow{2}{*}{$\begin{array}{l}\text { Aerobic } \\
22.43 \pm 0.61\end{array}$} & \multirow{2}{*}{$\begin{array}{l}\text { Anaerobic } \\
21.8 \pm 1.29\end{array}$} & \multirow{2}{*}{$\begin{array}{l}\text { Aerobic } \\
20.07 \pm \\
1.46 \\
\end{array}$} & \multirow{2}{*}{$\begin{array}{l}\text { Anaerobic } \\
17.83 \pm \\
1.43\end{array}$} & \multirow{2}{*}{$\begin{array}{l}\text { Aerobic } \\
17.27 \pm 0.90\end{array}$} & \multirow{2}{*}{$\begin{array}{l}\text { Anaerobic } \\
15.26 \pm 1.15\end{array}$} \\
\hline ED1 & F-BG 1.5\% & & & & & & \\
\hline ED2 & F-BG 4\% & $25.10 \pm 0.69$ & $\begin{array}{l}23.23 \pm \\
1.45\end{array}$ & $\begin{array}{l}21.90 \pm \\
1.51\end{array}$ & $\begin{array}{l}20.16 \pm \\
0.84\end{array}$ & $17.84 \pm 1.03$ & $16.76 \pm 0.88$ \\
\hline$C D$ & SMFP $0.76 \%$ & $29.83 \pm 0.23$ & $\begin{array}{l}29.83 \pm \\
1.31\end{array}$ & $\begin{array}{l}25.83 \pm \\
1.31\end{array}$ & $26.5 \pm 1.47$ & $21 \pm 0.81$ & $20.73 \pm 0.90$ \\
\hline \multicolumn{8}{|c|}{ ZOIs against $L$. casei } \\
\hline \multicolumn{2}{|c|}{ Experimental } & Microaerophilia & Anaerobic & $\begin{array}{l}\text { Micro- } \\
\text { aerophilia }\end{array}$ & Anaerobic & Microaerophilia & Anaerobic \\
\hline ED1 & F-BG $1.5 \%$ & $10.5 \pm 0.41$ & $\begin{array}{l}11.17 \pm \\
1.02\end{array}$ & $\begin{array}{l}8.17 \pm \\
0.62\end{array}$ & $6.5 \pm 0.41$ & $7.0 \pm 0.82$ & $5.83 \pm 0.24$ \\
\hline ED2 & F-BG $4 \%$ & $13.1 \pm 0.54$ & $\begin{array}{l}15.33 \pm \\
0.85\end{array}$ & $\begin{array}{l}10.17 \pm \\
1.03\end{array}$ & $11.0 \pm 0.816$ & $8.67 \pm 0.47$ & $8.83 \pm 0.62$ \\
\hline$C D$ & SMFP $0.76 \%$ & $16.33 \pm 1.25$ & $\begin{array}{l}17.67 \pm \\
0.47\end{array}$ & $\begin{array}{l}11.33 \pm \\
0.62\end{array}$ & $\begin{array}{l}14.33 \pm \\
0.47\end{array}$ & $10.4 \pm 0.43$ & $11.33 \pm 0.47$ \\
\hline
\end{tabular}

Abbreviation: ZOI, zone of inhibition. 
formulation can enhance its potential for converting tooth surface hydroxyapatite to more acid-resistant fluorohydroxyapatite and its ability to inhibit bacterial enzymes. ${ }^{20,21}$ For apatite formation in teeth, the F-nBG particles must stay on the teeth and their surroundings for sufficient time to induce remineralization without undergoing dilution by saliva and beverage intake. As salivary flow is lowest at night during sleep, the desired time for apatite formation should be less than 8 hours $^{22}$ following the use of dentifrices enriched with F-nBG fillers.

For efficacy of fluoride dentifrice, free available fluoride in an appropriate concentration is required. This means that the fluoride agent in dentifrice should not chemically react with any other dentifrice ingredients. ${ }^{23}$ Fluoride compounds generating fluoride ions like sodium fluoride, stannous fluoride, and ammonium fluoride are not compatible with chalkbased abrasives as ionic fluoride reacts with ionic calcium forming insoluble calcium fluoride. ${ }^{23,24}$ By contrast, sodium monofluorophosphate [SMFP $\left(\mathrm{Na}_{2} \mathrm{PO}_{3} \mathrm{~F}\right)$ ] displays greater compatibility with calcium-based abrasives, as the monofluorophosphate anion is stable and therefore cannot bind to ionic calcium. ${ }^{23}$ In the mouth, protective action in SMFP dentifrices results either by the direct effect of the monofluorophosphate anions or by the fluoride ions released due to hydrolytic degradation of SMFP in the mouth. ${ }^{25,26}$

Studies have shown that SMFP dentifrices with calcium-based abrasives lose an average of $25 \%$ to $35 \%$ ionic fluoride upon aging at $22^{\circ} \mathrm{C}$ and $29^{\circ} \mathrm{C}$, respectively, within 1 year. ${ }^{27-29} \mathrm{Although}$ the commercial dentifrices used in this study were within 3 years of expiration, high-temperature storage conditions (before coming to laboratory) could be blamed for the presence of smaller amounts of free fluoride available in the elutes and suspensions of the control group.

The literature mostly focuses on the total fluoride content of dentifrices and does not deliver sufficient information about the effective concentrations of free available fluoride in toothpaste to yield best anticaries effectiveness. The guidelines for cosmetic dentifrices mostly focus on total fluoride content only and state that this should not surpass 1500 ppm fluoride. The US Food and Drug Administration states that NaF/SMFP dentifrices comprising total fluoride of 850 to $1150 \mathrm{ppm}$ should hold $\geq 65 \mathrm{ppm}$ and $\geq 800 \mathrm{ppm}$ free fluoride, respectively. The justification for these values remains unclear. ISO Standard $11609^{30}$ at present mentions requirements for the physical and chemical properties of dentifrices, provides guidelines for suitable test methods including total fluoride content of dentifrice, and does not report the necessity to specify the amount of free fluoride in dentifrice, although this is a vital condition for anticaries efficiency. ISO7405[1 $]^{31}$ specifies and quantitative requirements for freedom from biological and toxicological hazards; however, no specific ISO guidelines for microbiological studies available. In addition, not a single study was found covering fluoride release of dentifrice containing F-nBG fillers. As all the novel dentifrices contain calcium carbonate as an abrasive, therefore, the effect of ionic calcium on free available fluoride in elutes and suspensions of novel dentifrices cannot be established. This study not only highlights the fluoride release behavior of the novel dentifrices enriched with F-nBGs but also covers the antimicrobial analysis of such dentifrices.

There was a need to incorporate a bactericidal agent in the dentifrice. The partial dissolution of $\mathrm{ZnO}$ particles releases $\mathrm{Zn}^{2+}$ ions in an aqueous suspension. ${ }^{14} \mathrm{Zinc}$ ions $\left(\mathrm{Zn}^{2+}\right)$ display antimicrobial action against many bacterial strains through direct contact with cell walls. ${ }^{14}$ For this purpose, $\mathrm{ZnO}$ nano powder was incorporated. In this study, the methods include the content and intent of the recommended methodology of the American Microbiological Society (ASM). For antimicrobial analysis, slurries of all the included dentifrices were made in various dilutions, as the level at which antimicrobial properties are buffered or lost in dilution by saliva is important. Agar well diffusion tests revealed that inhibition zones decreased in a dose-dependent manner for the dilutions $1: 1$, $1: 2$, and $1: 4$ of dentifrice slurries, further supporting the direct proportionality of filler loading of F-nBG. No increase in zone size was observed after 48 hours. All tested dentifrices exhibited greater antibacterial activity against the major cariogenic pathogen, that is S. mutans, than L. casei. With regard to the evaluation of the facultative nature of $S$. mutans, the recorded diameters of inhibition zones in both aerobic and anaerobic conditions were similar, whereas the inhibition zones recorded for $L$. casei were larger in the case of anaerobic conditions when compared with the $\mathrm{CO}_{2}$ jar.

The inhibition zones observed for ED2 were greater than ED1 because of greater fluoride content along with $\mathrm{ZnO}$ and consequent fluoride release. The ZOIs for $\mathrm{CD}$ were slightly elevated when compared with the inhibition zone for ED2, most likely due to incorporation of anticalculus agent-tetrasodium pyrophosphate in the commercial dentifrice-for enhanced cavity protection. According to previous studies, some dentifrices containing such agents may provide reduction in biofilm formation by approximately $15 \%{ }^{32,33}$

MBC values of tested dentifrices revealed that all the test dentifrices had greater effectiveness against $S$. mutans compared with $L$. casei. It also revealed that $3 \mathrm{wt} \% \mathrm{ZnO}$ and an increased concentration of F-nBG directly corresponded to increased bactericidal potential of experimental dentifrices. $\mathrm{MBC}$ values for $\mathrm{ED} 2$ (4 wt\% F-nBG) were same as that for $\mathrm{CD}$ (Colgate Cavity protection). It is important to consider that MIC values are bacteriostatic, ${ }^{34}$ whereas $\mathrm{MBC}$ values are bactericidal as they corresponded to subcultured plates which showed absence of bacterial growth. ${ }^{35}$ Further, in vitro studies need to be performed to assess the tooth remineralization potentials of these dentifrices to gauge their efficacy in relieving enamel white spot lesions and dentin hypersensitivity. Finally, cytotoxicity testing and in vivo usage tests should be performed to introduce the most suitable composition commercially.

\section{Conclusion}

It is concluded that in dentifrices, mainly the composition of the fluoride source, governs fluoride release behavior. Ionic fluoride in elutes and suspensions of both novel dentifrices was directly proportional to F-nBG filler loading. Among novel dentifrices, maximum mean fluoride release 
was detected in elutes and suspensions of group ED2, which contained the highest filler loading, that is, $4 \mathrm{wt} \% \mathrm{~F}-\mathrm{nBG}$. Filler loading matched the fluoride release of the commercial dentifrice.

Statistical analysis indicated that ED2 (F-nBG 4 wt.\%) demonstrated similar antimicrobial potential to that of an ordinary, non-tetra sodium pyrophosphate-containing fluoride dentifrice in terms of ZOIs for both bacterial strains under all growth conditions. The MBC obtained for ED2 was also similar to obtained $\mathrm{MBC}$ values for $\mathrm{CD}$. Incorporation of F-nBG and $\mathrm{ZnO}$ provide a multi-benefit approach to simultaneously treating early white spot lesions, reducing bacterial growth, and providing core plaque control.

\section{Conflict of Interest}

None declared.

\section{References}

1 Cvikl B, Lussi A, Gruber R. The in vitro impact of toothpaste extracts on cell viability. Eur J Oral Sci 2015;123(3):179-185

2 Baglar S, Nalcaci A, Tastekin M. The effect of temperature change on fluoride uptake from a mouthrinse by enamel specimens. Eur J Dent 2012;6(4):361-369

3 Ricomini Filho AP, Tenuta LM, Fernandes FS, Calvo AF, Kusano SC, Cury JA. Fluoride concentration in the top-selling Brazilian toothpastes purchased at different regions. Braz Dent J 2012;23(1):45-48

4 Guven Y, Ustun N, Tuna EB, Aktoren O. antimicrobial effect of newly formulated toothpastes and a mouthrinse on specific microorganisms: an in vitro study. Eur J Dent 2019;13(2):172-177

5 Cury JA, Oliveira MJ, Martins CC, Tenuta LM, Paiva SM. Available fluoride in toothpastes used by Brazilian children. Braz Dent J 2010;21(5):396-400

6 Nascimento HARd. Ferreira JMS, Granville-Garcia AF, Costa EMMdB, Cavalcante ALA, Sampaio FC. Estimation of toothpaste fluoride intake in preschool children. Braz Dent J 2013;24(2):142-146

7 Missel EM, Cunha RF, Vieira AE, Cruz NV, Castilho FC, Delbem AC. Sodium trimetaphosphate enhances the effect of 250 p.p.m. fluoride toothpaste against enamel demineralization in vitro. Eur J Oral Sci 2016;124(4):343-348

8 Zafar MS, Khurshid Z, Najeeb S, Zohaib S, Rehman IU, Therapeutic applications of nanotechnology in dentistry. In: Andronescu E, Grumezescu A, eds. Nanostructures for Oral Medicine. Elsevier;2017:833-862

9 Erol-Taygun M, Zheng K, Boccaccini AR. Nanoscale bioactive glasses in medical applications. Int J Appl Glass Sci 2013;4:136-148

10 Curtis AR, West NX, Su B. Synthesis of nanobioglass and formation of apatite rods to occlude exposed dentine tubules and eliminate hypersensitivity. Acta Biomater 2010;6(9):3740-3746

11 Gul H, Zahid S, Zahid S, Kaleem M, Khan AS, Shah AT. Sol-gel derived fluoride-doped bioactive glass powders: Structural and long-term fluoride release/pH analysis. J Non-Cryst Solids 2018;498:216-222

12 Kanel SR, Al-Abed SR. Influence of $\mathrm{pH}$ on the transport of nanoscale zinc oxide in saturated porous media. J Nanopart Res 2011;13:4035-4047

13 Takatsuka T, Tanaka K, Iijima Y. Inhibition of dentine demineralization by zinc oxide: in vitro and in situ studies. Dent Mater 2005;21(12):1170-1177
14 Pasquet J, Chevalier Y, Pelletier J, Couval E, Bouvier D, Bolzinger MA. The contribution of zinc ions to the antimicrobial activity of zinc oxide. Colloids Surf A Physicochem Eng Asp 2014;457:263-274

15 Ahtzaz S, Nasir M, Shahzadi L, et al A study on the effect of zinc oxide and zinc peroxide nanoparticles to enhance angiogenesis-pro-angiogenic grafts for tissue regeneration applications. Mater Des 2017;132:409-418

16 Pearce EI. A laboratory evaluation of New Zealand fluoride toothpastes. N Z Dent J 1974;70(320):98-108

17 Balouiri M, Sadiki M, Ibnsouda SK. Methods for in vitro evaluating antimicrobial activity: a review. J Pharm Anal 2016;6(2):71-79

18 Inetianbor J, Ehiowemwenguan G, Yakubu J, Ogodo A. In-vitro antibacterial activity of commonly used toothpastes in Nigeria against dental pathogens. J Adv Sci Res 2014;2:40-45

19 DeSchepper EJ, White RR, von der Lehr W. Antibacterial effects of glass ionomers. Am J Dent 1989;2(2):51-56

20 Featherstone JD. Prevention and reversal of dental caries: role of low level fluoride. Community Dent Oral Epidemiol 1999;27(1):31-40

21 Selwitz RH, Ismail AI, Pitts NB. Dental caries. Lancet 2007;369(9555):51-59

22 Rabiee SM, Nazparvar N, Azizian M, Vashaee D, Tayebi L. Effect of ion substitution on properties of bioactive glasses: a review. Ceram Int 2015;41:7241-7251

23 Benzian H, Holmgren C, Buijs M, van Loveren C, van der Weijden F, van Palenstein Helderman W. Total and free available fluoride in toothpastes in Brunei, Cambodia, Laos, the Netherlands and Suriname. Int Dent J 2012;62(4):213-221

24 Ericsson Y. Fluorides in dentifrices. Investigations using radioactive fluorine. Acta Odontol Scand 1961;19:41-77

25 Ingram GS. The reaction of monofluorophosphate with apatite. Caries Res 1972;6(1):1-15

26 Grøn P, Brudevold F, Aasenden R. Monofluorophosphate interaction with hydroxyapatite and intact enamel. Caries Res 1971;5(3):202-214

27 Hashizume LN, Lima YB, Kawaguchi Y, Cury JA. Fluoride availability and stability of Japanese dentifrices. J Oral Sci 2003;45(4):193-199

28 Bardal PA, Olympio KP, da Silva Cardoso VE, de Magalhães Bastos JR, Buzalaf MA. Evaluation of total pH and soluble and ionic fluoride concentrations in dentifrices commercially available in Brazil. Oral Health Prev Dent 2003;1(4):283-289

29 Conde NC, Rebelo MA, Cury JA. Evaluation of the fluoride stability of dentifrices sold in Manaus, AM, Brazil. Pesquisa Odontol Bras 2003;17:247-253

30 ISO 11609. Dentistry - Dentifrices - Requirements, test methods and marking. 3rd ed., 2017-06

31 ISO 7405. Dentistry - Evaluation of biocompatibility of medical devices used in dentistry. 3rd ed., 2018-11

32 Grossman E, Hou L, Bollmer BW, et al. Triclosan/pyrophosphate dentifrice: dental plaque and gingivitis effects in a 6-month randomized controlled clinical study. J Clin Dent 2002;13(4):149-157

33 Pedrazzi V, Corsi LP, Pedrazzi H, Netto EI, Nascimento Cd, Issa JP. Clinical evaluation of residual tetrasodium pyrophosphate released from two different anticalculus flosses. Braz Dent J 2015;26(2):116-120

34 Aamer A, Abdul-Hafeez M, Sayed S. Minimum inhibitory and bactericidal concentrations (MIC and MBC) of honey and bee propolis against multi-drug resistant (MDR) Staphylococcus sp. isolated from bovine clinical mastitis. Altern Integr Med 2014;3(4):1000171

35 Moran J, Addy M, Wade W. Determination of minimum inhibitory concentrations of commercial toothpastes using an agar dilution method. J Dent 1988;16(1):27-31 